\title{
Der Minsker Prozess: Gesellschaftliche Wahrnehmungen des Konfliktes in der Ostukraine
}

\author{
Cécile Druey, Anna Hess, Julia Kaplan, Valentina Cherevatenko"
}

\begin{abstract}
Der Minsker Prozess, also die Verhandlungen zur Lösung des Konflikts in der Ostukraine, hat seit seinem Beginn 2014 kaum konkrete Erfolge vorzuweisen. Interviews mit Menschen aus der Ukraine und Russland zeigen, wie die vom Konflikt unmittelbar Betroffenen den Minsker Prozess wahrnehmen. Die vorliegende Analyse legt den Fokus auf den Streit um die Wiederherstellung der Souveränität der Ukraine in den nicht von der Regierung kontrollierten Gebieten (NGCAs) der Regionen Donezk und Luhansk. Dabei werden zwei Hauptpositionen deutlich: „zuerst die Grenze“ und „zuerst der Status“. Werden die Bedürfnisse und Ängste untersucht, die diesen Positionen zugrunde liegen, lassen sich gemeinsame Interessen herausfiltern. So können schließlich Lösungen entwickelt werden, die für alle Seiten akzeptabel sind.
\end{abstract}

Schlagworte

Ukraine, Russland, Donbas, Minsker Prozess, Verhandlungen

Bitte zitieren als: Cécile Druey, Anna Hess, Julia Kaplan, Valentina Cherevatenko, Der Minsker Prozess: Gesellschaftliche Wahrnehmungen des Konfliktes in der Ostukraine, OSCE Insights 8 (Baden-Baden: Nomos, 2021), unter: https://doi.org/10.5771/9783748911630-08

\section{Einleitung}

Seit 2014 gibt es Versuche zur Konfliktbewältigung im Donbas-Gebiet in der Ostukraine, den sogenannten Minsker Prozess. Er soll die Minsker Vereinbarungen umsetzen, um Frieden und Sicherheit in der Ostukraine wiederherzustellen. Dieses Ziel ist jedoch nach wie vor außer Reichweite. Kern des Problems sind die sehr unterschiedlichen Ansichten über zentrale Bestimmungen auf Seiten der Offiziellen bei den Verhandlungen sowie in der Bevölkerung der vom Konflikt betroffenen Gebiete.
Diese unterschiedlichen gesellschaftlichen Positionen sollen hier aufgezeigt und die damit verbundenen Interessen,

* Dr. Cécile Druey

Universität Bern, Schweiz

cecile.druey@hist.unibe.ch

Anna Hess

Center for Security Studies

ETH Zürich, Schweiz

Dr. Julia Kaplan

National Institute for Strategic Studies (NISS)

Kiew, Ukraine

Dr. Valentina Cherevatenko

Union of the Don Women

Novocherkassk, Russland 
Bedürfnisse und Ängste untersucht werden. Im Mittelpunkt der Analyse steht die Wahrnehmung des offiziellen Friedensprozesses und seiner wichtigsten Bestimmungen. Dabei geht es darum, gemeinsame Interessen aller Beteiligten zu finden, um den Weg zu einem nachhaltigen Frieden freizumachen.

Dieser Beitrag basiert auf einer zwischen 2017 und 2020 durchgeführten Studie ${ }^{1}$, die die Wahrnehmungen des Minsker Prozesses in verschiedenen Regionen der Ukraine, einschließlich der nicht von der Regierung kontrollierten Gebiete (NGCAs) in den Regionen Donezk und Luhansk, sowie in Russland analysierte. Diese Studie zeigte, dass die breitere Gesellschaft zwar aus dem Verhandlungsprozess in Minsk ausgeschlossen war, sich ihre Wahrnehmungen aber im Großen und Ganzen mit den widerstreitenden Positionen deckten, die auch in den Verhandlungen zu beobachten waren. Der vorliegende OSCE InsightsBeitrag analysiert die Interessen, die diesen gesellschaftlichen Wahrnehmungen zugrunde liegen, und identifiziert übereinstimmende Interessen. Eine solche Analyse schafft Raum, um Lösungen zu finden, die für alle Seiten akzeptabel sind, was nicht nur dem offiziellen Friedensprozess zugutekommt, sondern auch alternativen friedensstiftenden Initiativen.

Dieser Beitrag untersucht einen zentralen Aspekt des Minsker Prozesses: die Wiederherstellung der ukrainischen Staatlichkeit in der Ostukraine. Auch für die im Rahmen unserer Studie interviewten Personen gilt dieser Punkt als einer der strittigsten. Hierbei konn- ten wir zwei Hauptnarrative identifizieren, die wir „zuerst die Grenze“ und „zuerst der Status“ nannten. Eine genauere Untersuchung der Interessen, die diesen sich widersprechenden Narrativen zugrunde liegen, zeigte weitgehend übereinstimmende Ängste und Bedürfnisse von Gesprächspartner*innen auf verschiedenen Seiten. Die dringendsten Anliegen waren, besonders unter den Interviewten aus der unmittelbaren Nachbarschaft des Konflikts: Entmilitarisierung, physische Sicherheit, sozioökonomische Mobilität und politische Partizipation. Unsere Analyse zeigt, dass die gemäßigten Positionen innerhalb der beiden Hauptnarrative Raum für Kompromiss-Lösungen bieten.

Unsere Untersuchung beruht auf der Harvard-Methode der interessenbasierten Verhandlungen. Ein Schlüsselprinzip dieser Methode ist die Trennung der an einem Konflikt beteiligten Parteien und ihrer Positionen von den zugrunde liegenden Interessen. So erhöht das Verständnis für die Interessen, Bedürfnisse, und Ängste der Parteien die Chancen auf eine für alle Seiten akzeptable Lösung. ${ }^{2}$

Die Daten wurden in 144 qualitativen Interviews gesammelt, die zwischen 2018 und 2019 geführt wurden und alle geografischen Regionen abdeckten, die vom Konflikt im Donbas betroffen waren. Dazu gehören die von der Regierung kontrollierten Gebiete (GCAs) in der Ukraine (in den mittleren, westlichen, östlichen und südlichen Teilen des Landes), die NGCAs (in den Regionen Luhansk und Donezk) und Russland (die an die Ukraine angrenzende Region sowie die mittleren und nördlichen Teile Russ- 
lands). Zusätzlich wurden Interviews mit Personen aus zwei nicht-geografischen Kategorien geführt: Binnenvertriebene aus dem Donbas in der Ukraine sowie Flüchtlinge aus dem Donbas in Russland. Letztere erwiesen sich als wertvolle Quelle, da die Positionen, die von Flüchtlingen aus dem Donbas in Russland vertreten werden, oftmals die Meinungen der Bewohner*innen der NGCAs offener wiedergeben. ${ }^{3}$

\section{Der Minsker Prozess}

Seit seinem Beginn 2014 hat der Konflikt in der Ostukraine über 13.000 Menschenleben gefordert. ${ }^{4}$ Die Ukraine beherbergt etwa 1,5 Millionen Binnenflüchtlinge, ${ }^{5}$ und mindestens eine Million Flüchtlinge haben den Donbas in Richtung Russland verlassen. ${ }^{6}$ Als Ergebnis des Krieges verlor die Ukraine die Kontrolle über ihre Staatsgrenze zu Russland sowie Teile des Donbas, nämlich die selbst ernannten Volksrepubliken Donezk und Luhansk. Eine 472 Kilometer lange Kontaktlinie unterteilt die Region in GCAs und NGCAs. ${ }^{7}$ Der Konflikt hat nicht nur die staatliche Souveränität der Ukraine nachhaltig geschwächt, sondern auch massive Schäden an der Infrastruktur hinterlassen. Als Folge davon ist die Wirtschaft zusammengebrochen, und die betroffene Bevölkerung lebt unter schwierigsten humanitären Bedingungen. Die Sicherheitssituation entlang der Kontaktlinie ist nach wie vor instabil. Die Konfliktlinien folgen jedoch nicht geografischen und ideologischen Kriterien, sondern verlaufen mitten durch die Gesellschaft, sodass sich Familienmitglieder und Freunde oft in verschiedenen Lagern finden.

Internationale Bemühungen um eine Lösung des Konflikts in der Ostukraine gibt es seit 2014, aber die Gewalt ließ erst Ende 2016/Anfang 2017 nach. Gemeinhin werden als Minsker Vereinbarungen drei Dokumente von September 2014 (das Protokoll von Minsk und das Memorandum von Minsk) und Februar 2015 (das Maßnahmenpaket zur Umsetzung der Minsker Vereinbarungen) bezeichnet, die von Russland, der Ukraine und Vertretern der NGCAs unterzeichnet wurden, unter der Schirmherrschaft der vier Staats- und Regierungschefs des Normandie-Formats (Deutschland, Frankreich, Russland und die Ukraine). Die Vereinbarungen dienen als Grundlage für Gespräche mit der trilateralen Kontaktgruppe der OSZE (TKG) und ihrer vier thematischen Arbeitsgruppen. Der Minsker Prozess, und hier besonders die TKG, ist das einzige Format, das alle am Konflikt beteiligten Parteien auf offizieller Ebene zusammenbringt. Jedoch gibt es auch sechs Jahre nach Unterzeichnung der Vereinbarungen und trotz der alle zwei Wochen stattfindenden Treffen in Minsk weder einen umfassenden und anhaltenden Waffenstillstand noch eine politische Lösung. Ein entscheidendes Hindernis bei der Umsetzung der Minsker Vereinbarungen ist nach wie vor die Frage der Sequenzierung. Weitere Hürden sind unter anderem die fehlende Transparenz bei den Abläufen und die kontroverse politische und militärische Rolle Russlands. 
Die Wiederherstellung der ukrainischen Staatlichkeit: Von entgegengesetzten Positionen zu gemeinsamen Interessen?

Wie oben erwähnt ist die Wiederherstellung der ukrainischen Staatlichkeit in der Ostukraine ein Hauptstreitpunkt des Minsker Prozesses. Die ukrainische Regierung besteht darauf, dass zuerst die Kontrolle über die Grenze mit Russland wiederhergestellt werden muss, bevor man über den Status der NGCAs entscheiden kann. Die NGCAs wiederum fordern mit russischer Rückendeckung Autonomie und Sicherheitsgarantien, bevor über eine mögliche Wiedereingliede- rung der Gebiete in ukrainisches Territorium diskutiert werden kann. Auf den ersten Blick spiegeln die unterschiedlichen Narrative, die wir in unserer Studie ausgemacht haben und deren Ansätze von „zuerst die Grenze“ bis „zuerst der Status" reichen, die offiziellen Positionen, die zum gegenwärtigen Patt geführt haben. Unsere Untersuchung zeigt jedoch ein weites Spektrum von Positionen. Zwischen den Extremen „harte Reintegration“ und „vollständige Unabhängigkeit" gibt es auch gemäßigtere Ansichten, die Lösungen ermöglichen (siehe Tabelle 1).

\section{Tabelle 1: Überblick über Narrative und Positionen}

\begin{tabular}{|c|c|c|c|}
\hline Narrativ & Position & $\begin{array}{l}\text { Zugrunde liegende } \\
\text { Ängste/Interessen }\end{array}$ & $\begin{array}{l}\text { Geteilt von (Gruppe von } \\
\text { Befragten) }\end{array}$ \\
\hline \multirow[t]{3}{*}{$\begin{array}{l}\text { Narrativ I } \\
\text { „zuerst die Grenze“: } \\
\text { Wiederherstellung } \\
\text { ukrainischer Kon- } \\
\text { trolle hat oberste } \\
\text { Priorität }\end{array}$} & $\begin{array}{l}\text { Position } 1 \\
\text { „Unbedingte Reintegration und } \\
\text { Wiederherstellung des Status } \\
\text { quo ante“ }\end{array}$ & $\begin{array}{l}\text { Angst: } \\
\text { - } \quad \text { schleichende Russifi- } \\
\text { zierung der Ukraine } \\
\text { Interesse: } \\
\text { - Status quo ante (Ukrai- } \\
\quad \text { ne, einschließlich } \\
\text { Donbas und Krim) }\end{array}$ & $\begin{array}{ll}\text { - } & \text { Ukraine (vor allem } \\
\text { südliche und zentrale } \\
\text { Regionen) } \\
\text { - } \\
\text { Russland (zentrale } \\
\text { Regionen) }\end{array}$ \\
\hline & $\begin{array}{l}\text { Position } 2 \\
\text { „Territoriale Integrität und na- } \\
\text { tionales Interesse“ }\end{array}$ & $\begin{array}{l}\text { Angst: } \\
\text { - } \quad \text { Sonderstatus der } \\
\text { NGCA als Bedro- } \\
\text { hung des ukraini- } \\
\text { schen Staats (territo- } \\
\text { riale Fragmentierung) }\end{array}$ & $\begin{array}{ll}- & \text { Ukraine (gesamt) } \\
\text { - } & \text { Russland (gesamt) }\end{array}$ \\
\hline & $\begin{array}{l}\text { Position } 3 \\
\text { "Sanfte Reintegration und Wie- } \\
\text { derherstellung der Staatlichkeit" }\end{array}$ & $\begin{array}{l}\text { Interessen: } \\
\text { - } \quad \text { sozioökonomisches } \\
\text { Überleben } \\
\text { - Wiederherstellung } \\
\text { der Staatlichkeit und } \\
\text { der Infrastruktur im } \\
\text { Konfliktgebiet }\end{array}$ & $\begin{array}{l}\text { Ukraine, Gebiete na- } \\
\text { he der Konfliktzone } \\
\text { (GCAs und NGCAs) } \\
\text { Russland (Grenzge- } \\
\text { biet) }\end{array}$ \\
\hline
\end{tabular}




\begin{tabular}{|c|c|c|c|}
\hline Narrativ & Position & $\begin{array}{l}\text { Zugrunde liegende } \\
\text { Ängste/Interessen }\end{array}$ & $\begin{array}{l}\text { Geteilt von (Gruppe von } \\
\text { Befragten) }\end{array}$ \\
\hline \multirow[t]{2}{*}{$\begin{array}{l}\text { Narrativ II } \\
\text { „Zuerst der Status“: } \\
\text { Sonderstatus/ Auto- } \\
\text { nomie/ } \\
\text { Unabhängigkeit für } \\
\text { die NGCAs hat } \\
\text { oberste Priorität }\end{array}$} & $\begin{array}{l}\text { Position } 4 \\
\text { „Unabhängigkeit oder Vereini- } \\
\text { gung mit Russland“ }\end{array}$ & $\begin{array}{l}\text { Ängste: } \\
\text { - Isolation } \\
\text { •harte Integration“ } \\
\text { als humanitäre Kata- } \\
\text { strophe für die Be- } \\
\text { wohner*innen der } \\
\text { NGCAs } \\
\text { Interesse: } \\
\text { - Menschliche Sicher- } \\
\text { heit in den NGCAs }\end{array}$ & $\begin{array}{ll}- & \text { NGCAs } \\
\text { - } & \text { Flüchtlinge aus dem } \\
& \text { Donbas in Russland }\end{array}$ \\
\hline & $\begin{array}{l}\text { Position } 5 \\
\text { „Übergangsweise Autonomie } \\
\text { und sanfte Unabhängigkeit“ }\end{array}$ & $\begin{array}{l}\text { Interessen: } \\
\text { - Menschliche Sicher- } \\
\text { heit in den NGCAs } \\
\text { Wiederherstellung } \\
\text { der Staatlichkeit und } \\
\text { der Infrastruktur im } \\
\text { Konfliktgebiet }\end{array}$ & $\begin{array}{ll}\text { - } & \text { Ukraine GCAs (ge- } \\
\text { samt) } \\
\text { - } \\
\text { - } \\
\text { NGCAssland (Grenzge- } \\
\text { biet) }\end{array}$ \\
\hline
\end{tabular}

Narrativ I: Reintegration und „zuerst die Grenze“"

Die Spannung zwischen den beiden Hauptnarrativen „zuerst die Grenze“ und „zuerst der Status“ findet ihre Entsprechung in der Herausforderung, eine Einigung über die Sequenzierung der Umsetzung der Minsker Vereinbarungen zu erreichen. Die Befragten, die „zuerst die Grenze" befürworten, glauben, dass die Ukraine als Erstes die Kontrolle über die Grenze zu Russland wiedererlangen muss, bevor weitere Schritte erfolgen können.

\section{Position 1: „Unbedingte Reintegration und Wiederherstellung des Status quo ante"}

„Ich bin nicht für irgendeinen Sonderstatus für diese Gebiete [die NGCAs], außer, dass diese Gebiete strikter Disziplin, Überwachung und Ordnung unterworfen sein sollten. Kurz gesagt, ich will und halte es auch für fair und richtig, dass diese Gebiete bestraft werden." (Person aus der Zentralukraine)

Der Ruf nach einer ,harten Reintegration" der NGCAs in ukrainisches Territorium gehört zu den radikaleren Positionen, oft im Verbund mit der Forderung nach Wiederherstellung des territorialen Status quo ante (einschließlich der Krim). Diese "harte Reintegration“ beinhaltet für die Befragten die Wiedererlangung der 
vollständigen Kontrolle über die Grenze zu Russland, die völlige Schließung derselben und eine militärische Besetzung des Donbas durch ukrainische Streitkräfte. Sozioökonomisch würde dies die Wiederherstellung völliger Kontrolle über die Bevölkerung in den NGCAs bedeuten und deren „(Re-)Ukrainisierung“, wobei oftmals ein Wunsch nach Vergeltung mitschwingt, wie das obige Zitat zeigt. Die Option, den NGCAs auf der Grundlage des Sonderstatusrechts Autonomie zuzugestehen, wie in den Minsker Vereinbarungen diskutiert, wird von diesen Befragten strikt abgelehnt. Befürchtet wird, dass dieser Sonderstatus von Russland - über ein politisches Mitspracherecht der Entscheidungsträger im Donbas - dazu benutzt werden könnte, Druck auf Kiew auszuüben. ${ }^{8}$ Im Allgemeinen halten Anhänger*innen dieser Position die russische Aggression für den wichtigsten, wenn nicht gar einzigen Grund für den Konflikt im Donbas. Sie suchen ihr Heil im populären Motto „Russland raus!" und glauben, die Entrussifizierung des Donbas sei eine essenzielle Voraussetzung für den Frieden. Interessanterweise wird die Position "harte Reintegration“ und „Russland raus!“ nicht nur von Befragten in den südlichen und in zentralen Regionen der Ukraine vertreten, sondern auch von Befragten in Russland, vor allem in den Regionen, die am weitesten vom unmittelbaren Konfliktgebiet entfernt liegen.

Der Wunsch nach einer militärischen Lösung steht im Gegensatz zu der Sichtweise der Bewohner*innen der NGCAs und der Flüchtlinge aus dem Donbas in Russland. Er unterschiedet sich auch von den Meinungen der Befragten nahe der Konfliktzone im von Kiew kontrollierten Teil des Donbas und in Südrussland. Diese argumentieren, dass eine militärische Zurückeroberung des Donbas die Sicherheit und die wirtschaftliche Entwicklung der gesamten Region bedrohen würde.

Die radikale Position der "harten Reintegration" lässt sich zum Teil auf einen unzureichenden und falschen Informationsstand zu Inhalt und Abläufen des Minsker Prozesses zurückführen. Mehrere Befragte aus den GCAs und den NGCAs, unter den Donbas-Flüchtlingen und aus Russland geben an, wenige oder keine Informationen zum Friedensprozess zu haben. Und wenn sie diese doch erhalten, dann meist durch informelle Kanäle. Dabei halten sie Blogger und einflussreiche Eliten für besonders vertrauenswürdige Quellen. Dadurch werden Konzepte wie „Sonderstatus“, „Föderalismus“, „Autonomie“, und „Amnestie“ mit Mythen von Gebietsverlusten und schleichender Russifizierung assoziiert. Das Fehlen von Transparenz und verlässlicher Information über die Modalitäten dieser Schlüsselkonzepte hat zu Ängsten und zu einer Verhärtung der Positionen geführt.

\section{Position 2: „Territoriale Integrität und nationales Interesse"}

„[Die Wiederherstellung des Friedens bedeutet] für die Ukraine das Erreichen der vollständigen Kontrolle über die gesamte Staatsgrenze der Ukraine. Ich halte dies für absolut notwendig. Jedes Land hat das Recht 
auf Souveränität!“ (Person aus der Zentralukraine)

Diese Position betrachtet die Reintegration eher von einem rechtlichen und institutionellen als von einem ideologischen Standpunkt. Ihre Vertreter*innen glauben, die NGCAs sollten wieder eingegliedert und die Grenzkontrollen wiederhergestellt werden - entsprechend dem Recht und der Verpflichtung des ukrainischen Staats, die Rechtshoheit über sein gesamtes international anerkanntes Territorium auszuüben.

Der Standpunkt unterscheidet sich vom Ansatz der „harten Integration“ nicht nur durch Nüchternheit, sondern ruft auch weniger Widerspruch hervor. Unter den Befragten aus allen Gruppen, einschließlich Einwohnern*innen von NGCAs und den sogar noch radikaleren Flüchtlingen im Süden Russlands, herrscht ein breiter Konsens darüber, dass territoriale Integrität und ein intaktes Grenzregime mit Russland im Prinzip positiv zu betrachten seien, da sie die Grundvoraussetzungen für einen funktionsfähigen ukrainischen Staat bilden.

Wenn wir die Position „territoriale Integrität und nationales Interesse" und den radikaleren Ansatz der "harten Reintegration“ und „Russland raus!“ genauer betrachten, zeigt sich deutlich, dass beiden Positionen ein gemeinsames Hauptinteresse zugrunde liegt: das Bedürfnis nach ukrainischer Souveränität und die Angst vor einer weiteren Zersplitterung des ukrainischen Territoriums. Die Angst vor einer Fragmentierung der ukrainischen Staatsmacht geht Hand in Hand mit der Empörung über
Russlands Anspruch auf eine regionale Vormachtstellung. Der Ruf nach einem Rückzug Russlands und die zögerliche Zustimmung zu lokalen Wahlen und zu einem Sonderstatus für die NGCAs werden durch die Angst vor einer zunehmenden Russifizierung der Ostukraine befeuert. Zudem äußerten einige der Befragten aus Südrussland und dem von Kiew kontrollierten Teil des Donbas Besorgnis über die Geopolitisierung des Konflikts, insbesondere gegen die Instrumentalisierung des Donbas und die Durchlässigkeit seiner Grenze zu Russland. Denn genau diese ermöglicht es Moskau, den Konflikt entsprechend der jeweiligen $\mathrm{Hal}$ tung Kiews einzufrieren oder zu befeuern.

\section{Position 3: "Sanfte Reintegration und Wiederherstellung der Staatlichkeit"}
„[Die Kontrolle der Staatsgrenze durch die Ukraine bedeutet] Kontrol- le über die Ein- und Ausreise norma- ler Bürger*innen und Besucher*in- nen des Landes. Dasselbe gilt für die Beförderung von Gütern. Aber darüber reden sie [die Protokolle von Minsk] nicht, sie reden nur über das Verbot von Nachschub für die be- waffneten Streitkräfte..." (Person aus der Ostukraine/GCAs im Donbas)

Die Befürworter*innen einer „sanften Reintegration" bilden eine der moderateren Gruppen. Sie betonen nicht die Kontrolle oder Schließung der Grenze, sondern deren Wiederherstellung. Die Grenze soll ihre normale Funktion erfüllen, als Teil 
einer Wiederherstellung und Konsolidierung ukrainischer Staatlichkeit in den vom Konflikt unmittelbar betroffenen Zonen.

Diese Haltung deckt sich mit der von Johan Galtung formulierten Idee eines „positiven Friedens“. Dieser geht vom Ziel einer Stabilität jenseits rein militärischer Überlegungen aus und berücksichtigt nicht nur unmittelbare Probleme der (physischen) Sicherheit, sondern auch die Grundursachen des Konflikts. ${ }^{9}$ Die Befragten aus Gebieten nahe der Konfliktzone auf der ukrainischen Seite sowie in Südrussland betonen die Bedeutung einer „positiven“ staatlichen Präsenz als Grundlage einer erfolgreichen zukünftigen Wiedereingliederung. In ihren Augen beinhaltet dies die Wiederherstellung eines intakten Rechtssystems und einer funktionierenden sozioökonomischen Infrastruktur sowie das Vorhandensein administrativer Strukturen in der gesamten Ukraine, einschließlich der NGCAs. Danach gehört zur Wiederherstellung der ukrainischen Kontrolle über die Grenze nicht nur die Kontrolle über Waffen, sondern auch über die Beförderung von Personen und Gütern. Diese Sichtweise ist besonders verbreitet unter den Befragten aus Gebieten nahe der Konfliktzonen (d.h. aus dem von der Regierung kontrollierten Teil des Donbas, den NGCAs sowie Südrussland). Für sie sind Sicherheit und Mobilität an der ukrainisch-russischen Grenze und an der internen Kontaktlinie in der Ukraine besonders wichtig.

\section{Gemeinsame Interessen:}

Sozioökonomisches Überleben und

\section{Mobilität}

Die Bedürfnisse, die den Ansätzen für eine Wiederherstellung der Staatlichkeit und „zuerst die Grenze“ zugrunde liegen, sind mit Sicherheit, dem sozioökonomischen Überleben, Mobilität und einer würdevollen Existenz verknüpft. Viele Befragte aus NGCA-Regionen teilen dieses Interesse an einem „positiven Frieden“ und einer Lösung für das Problem der Wiedereingliederung, die den Menschen in den Mittelpunkt stellt. Sie äußerten Toleranz oder sogar Unterstützung für die Idee der Wiedereingliederung - unter der Bedingung, dass ihre Sicherheit garantiert und ihre sozioökonomischen Bedürfnisse gedeckt würden.

Die Wiederherstellung einer „positiven" staatlichen Präsenz der Ukraine im gesamten Donbas ist das zweite gemeinsame Interesse, das von Befragten aus Gruppen beiderseits der Grenze genannt wurde und Kompromissmöglichkeiten eröffnet.

Narrativ II: Autonomie und „zuerst der Status“

Die Anhänger*innen des zweiten Hauptnarrativs, „zuerst der Status“, betrachten die Gewährung eines Sonderstatus für die NGCAs (Unabhängigkeit, Autonomie oder Föderalisierung) mit anschließenden Lokalwahlen als die wichtigste Priorität. Die Gewährung eines solchen Status muss aus ihrer Sicht vor einer Wiederherstellung der Grenzkontrolle erfolgen und 
ist die Voraussetzung für weitere Schritte in Richtung Frieden. Wie der Ansatz „zuerst die Grenze“ stellt auch dieses Narrativ keine einheitliche Position mit Bezug auf die potenzielle Wiederherstellung der ukrainischen Staatlichkeit dar. Während einige „zuerst der Status“-Befürworter*innen auf dem Prinzip „Unabhängigkeit um jeden Preis“ für die NGCAs beharren, sind andere dafür, Lugansk und Donezk nur temporär einen Sonderstatus zu gewähren bevor eine umfassende Lösung gefunden werden kann. Auch hier bieten die moderaten Positionen Raum für die Sondierung von mehrheitsfähigen Lösungen.

\section{Position 4: „Unabhängigkeit oder Vereinigung mit Russland“"}

„Das Wort ,Ukraine“ bedeutet ,Tod mit Zöpfen' [...]. Ich meine, diese ganzen Verhandlungen sind ein Spiel mit dem Feuer. Macht, was ihr wollt. Erzählt mir im Radio und im Fernsehen, was immer ihr wollt. Mit einem freundlichen Lächeln erwürgt ihr euer eigenes Volk...“ (Donbas-Flüchtling in der Russischen Föderation)

Die Unterstützer der Haltung „Unabhängigkeit um jeden Preis" betrachten die mögliche Wiedereingliederung und Wiederherstellung der Kontrolle Kiews über die Grenze zu Russland als unmittelbare Bedrohung ihrer Sicherheit. Einige NGCA-Bewohner"innen sowie die meisten Donbas-Flüchtlinge in Russland meinen, dass „der Krieg an einem Punkt angekommen ist, von dem es kein Zurück mehr gibt“. Nach sechs Jahren Krieg und Entfremdung sei die maximale Unabhängigkeit von der zentralen Staatsmacht in Kiew die beste Option für die NGCAs. Falls die Unabhängigkeit nicht erreicht werden kann, ist auch eine Eingliederung in den russischen Staat für diese Gruppe eine mögliche Alternative.

Mit anderen Worten: Ebenso wie bei den radikalen Positionen innerhalb des Narrativs „zuerst die Grenze“ geht es bei der Haltung „Unabhängigkeit um jeden Preis" vor allem um die Rolle Russlands. Die Wahrnehmung Russlands als Garant des Friedens und selbstlose Schutzmacht der lokalen Bevölkerung ist hier verbreitet. Demnach gilt die potenzielle Vereinigung der NGCAs mit Russland als attraktive Alternative zur Wiedervereinigung mit der Ukraine, vor allem zur ,harten Wiedereingliederung“.

Die Haltung „Unabhängigkeit um jeden Preis“ entspringt dem unmittelbaren Bedürfnis der Betroffenen nach physischer und sozialer Sicherheit. Die Durchlässigkeit der Grenze zu Russland hat sich während der Kämpfe und der anschließenden Isolation von der Ukraine für die NGCA-Bewohner"innen als lebenswichtig erwiesen. Diese Befragten fürchten, dass sie zwischen einer abgeriegelten Außengrenze mit Russland und einem restriktiven Kontrollregime an der Kontaktlinie festsitzen könnten, falls keine Lösung für ihren politischen Status gefunden und keine Garantien von Kiew für die Sicherheit des Donbas gegeben werden sollten.

Der Ruf nach voller Unabhängigkeit ist nicht nur durch harte Sicherheitsinteressen, sondern auch durch psychosoziale 
Faktoren motiviert. Insbesondere fürchten die Bewohner*innen der NGCAs und die Donbas-Flüchtlinge in Russland im Falle einer Wiedereingliederung eine "Vergeltung“ der Ukraine. Diese Furcht hat sich durch die psychosozialen Auswirkungen des bewaffneten Konflikts und der jahrelangen Entfremdung zwischen den NGCAs und den GCAs noch weiter verstärkt. Insbesondere die Flüchtlinge aus dem Donbas in Russland, die durch die Kriegserfahrung schwer traumatisiert sind, fürchten, dass der Konflikt wieder aufflammen könnte, falls die Ukraine die volle Kontrolle über die NGCA-Gebiete wiedererlangt. Viele Einwohner*innen der NGCAs fürchten, mit einer Wiedereingliederung würden sie wegen angeblicher kultureller und linguistischer Unterschiede, die sich auch in ihrer gesellschaftspolitischen Ausrichtung (z.B. proMaidan oder anti-Maidan, pro-westlich oder pro-russisch) niederschlagen, von den GCAs als „Bürger zweiter Klasse“ behandelt.

Die Anhänger*innen dieser Position begründen die Notwendigkeit einer vollen Unabhängigkeit und Trennung vom ukrainischen Staat auch mit sozioökonomischen Argumenten. Der eingeschränkte Waren- und Personenverkehr, der fehlende Zugang zu wirtschaftlichen Möglichkeiten jenseits der Kontaktlinie und das Embargo gegen die NGCAs lassen die Aussichten für eine zukünftige lokale Entwicklung und Zusammenarbeit ungünstig erscheinen. Aus diese Wahrnehmung resultiert der Glaube, dass die einzigen praktikablen Optionen die Unabhängigkeit oder eine Vereinigung mit Russland sind. Auch diese Ängste sind direkt mit dem Bedürfnis nach Sicherheit und einem menschenwürdigen Leben verknüpft.

Ähnlich wie bei der „harten Wiedereingliederung" scheint die strikte Haltung „Unabhängigkeit oder Vereinigung mit Russland" zum Teil auf einem Mangel an Transparenz und Informationen über den Minsker Prozess und seine Bestimmungen $\mathrm{zu}$ beruhen. Eine bessere Kommunikationsstrategie würde deshalb allen Beteiligten helfen. ${ }^{10}$

\section{Position 5: „Übergangsweise Autonomie und sanfte Unabhängigkeit"}

\begin{abstract}
„Wenn es dort ein friedliches Leben gibt und fünf bis zehn Jahre vergehen, wird das Thema eines Sonderstatus mit der Zeit verschwinden." (Person aus der Zentralukraine)
\end{abstract}

Am anderen Ende des Spektrums innerhalb der Narrativs "zuerst der Status“ finden sich die Befürworter*innen der „übergangsweisen Autonomie“, die den Autonomiestatus für die NGCAs als einen wichtigen Schritt in Richtung Frieden sehen. Im Gegensatz zu den Vertreter*innen der zuvor genannten Haltung der ,vollen Unabhängigkeit oder Vereinigung mit Russland“ betrachten sie die Frage jedoch vom Standpunkt des nationalen Interesses der Ukraine. Die Befragten in dieser Gruppe sind insofern moderat, als sie akzeptieren, dass die Kriegserfahrung und jahrelange Entfremdung zwischen GCAs und NGCAs eine neue Realität geschaffen haben. Diese muss ihrer Ansicht nach im Friedensprozess 
berücksichtigt werden. Anstatt den Autonomiestatus als endgültige Lösung $\mathrm{zu}$ betrachten, verstehen sie ihn jedoch als vorübergehenden Kompromiss, welcher sicherstellen soll, dass die Ukraine "den Donbas nicht verliert“. Diese Befragten sehen die übergangsweise Autonomie, zusammen mit der Wiederherstellung der ukrainischen staatlichen Souveränität in den NGCAs, als einen der erfolgversprechendsten Wege zu einer Friedenslösung.

\section{Gemeinsame Interessen: Eine positive Präsenz des ukrainischen Staats, die den Bedürfnissen der Bevölkerung dient}

Mehrere der oben dargestellten Haltungen lassen zugrunde liegende gemeinsame Interessen erkennen. Die Position der „sanften Unabhängigkeit“ (Position 5) findet sich in den Antworten von Befragten aus allen Teilen der Ukraine wieder. Sie ist wie der Ansatz der „sanften Wiedereingliederung" eine moderate Haltung, die den Konflikt durch eine positive Präsenz des ukrainischen Staats lösen möchte, um die Bedürfnisse der Bevölkerung $\mathrm{zu}$ befriedigen, das nationale Interesse zu wahren und der zunehmenden Russifizierung des Donbas entgegenzuwirken.

Obwohl sich die Befürwortung der „übergangsweisen Autonomie mit sanfter Unabhängigkeit" am deutlichsten unter den Befragten aus den GCAs zeigte, scheint sie auch mit den Interessen der Bevölkerung in den NGCAs vereinbar zu sein. Ein Großteil der NGCA-Befragten war nicht strikt dagegen, dass ihr Gebiet
Teil der Ukraine bleiben (oder wieder werden) könnte. Vielmehr waren diese in erster Linie gegen eine "harte Wiedereingliederung“, und zwar aus Furcht vor ukrainischer Vergeltung.

\section{Empfehlungen}

Unsere Analyse der zwei wichtigsten Narrative zum Thema der Wiederherstellung ukrainischer Staatlichkeit hat übereinstimmende Interessen aufgedeckt, die Raum für einen potenziellen Dialog schaffen. Den meisten Haltungen liegen Interessen zugrunde, die mit dem Überleben, der Sicherheit, dem sozioökonomischen Wohlergehen und einer menschenwürdigen Existenz verbunden sind. Im folgenden Abschnitt bieten wir Empfehlungen für relevante Zielgruppen mit Bezug auf die von uns identifizierten gemeinsamen Anliegen. Jedoch würde eine detaillierte Ausformulierung konkreter Schritte den Rahmen dieses Beitrags sprengen. Diese Aufgabe muss von allen Beteiligten übernommen werden, die eine friedliche Lösung des Konflikts in der Ostukraine anstreben.

\section{1) Sicherheit und Überleben}

Vertreter*innen aller Gruppen nannten Sicherheit und ein Ende der Waffengewalt als Grundvoraussetzungen für ihre Existenz, für das Überleben des Staates und für die Wiederherstellung des Friedens. Die emotional aufgeladenen Hardliner*innen-Positionen „harte Wiederein- 
gliederung“ und „Unabhängigkeit um jeden Preis" beruhen auf tief sitzenden Empfindungen von Unsicherheit und existenzieller Bedrohung. In den Verhandlungen und Entscheidungen über die Wiederherstellung staatlicher Souveränität in den NGCAs müssen diese Ängste berücksichtigt werden, wenn eine Einigung über die Erneuerung der ukrainischen Staatlichkeit in den NGCAs möglich sein soll.

\section{Empfehlungen}

\section{a) an die ukrainische Regierung}

Die Wiedereingliederung von NGCAEinwohner*innen und rückkehrenden Flüchtlingen aus dem Donbas sollte erleichtert werden, z.B. durch Garantien, dass die Einwohner*innen der NGCAs in der Ukraine nicht diskriminiert werden.

\section{b) an die russische Regierung und die De-facto-Behörden in den NGCAs}

Die Entmilitarisierung und die Minenräumung in den NGCAs sollte aktiv unterstützt werden.

\section{2) Wiederherstellung der Staatlichkeit und einer positiven Präsenz des ukrainischen Staates}

Ein weiteres gemeinsames Interesse, das sich vor allem in den moderaten Positionen zeigt, ist die Wiederherstellung einer positiven Präsenz des ukrainischen Staates in der Region Donbas, die die Rechte der lokalen Bevölkerungen in den NGCAs und GCAs gleichermaßen respektiert und deren Bedürfnisse abdeckt. Den Befragten aus den Grenzregionen der GCAs, aus den NGCAs und aus Russland sind pragmatische Lösungen für Probleme bei der sozioökonomischen Entwicklung, dem Wiederaufbau und der grenzübergreifenden Mobilität wichtig. Viele Interviewte aus den NGCAs (in den Regionen Luhansk und Donezk) zeigten sich offen für die Idee einer „weichen Wiedereingliederung" auf der Basis eines Autonomiestatus, verbunden mit einer positiven Präsenz des ukrainischen Staates. Mehrere Befragte aus verschiedenen Gruppen sagten, die moderate Befürwortung der "weichen Wiedereingliederung" in Verbindung mit der Anerkennung einer übergangsweisen Autonomie oder "weicher Unabhängigkeit" sei ein gemeinsames Interesse, über das weiter diskutiert werden könne.

\section{Empfehlungen}

\section{a) an die ukrainische Regierung}

Es sollte eine umfassende und kohärente Strategie für den Staatsaufbau und die lokale Entwicklung in den NGCAs erarbeitet werden, um das Vertrauen und die Unterstützung der lokalen Bevölkerung zu gewinnen und eine Wiedereingliederung zu ermöglichen.

Es sollte eine unkomplizierte, sichere und transparente Regelung für den Austausch zwischen den NGCAs und GCAs 
an Checkpoints entlang der Kontaktlinie entwickelt werden.

\section{b) an die OSZE, die ukrainische Regierung und zivilgesellschaftliche Organisationen}

Zwischen der OSZE und dem Ministerium für die Wiedereingliederung der vorübergehend besetzten Gebiete der Ukraine sowie den zivilgesellschaftlichen Organisationen, die auf Übergangsjustiz, Dialoginitiativen und die lokale Entwicklung der NGCAs spezialisiert sind, sollten Mechanismen für eine Zusammenarbeit entwickelt werden.

\section{c) an die russische Regierung und die De- facto-Behörden in den NGCAs}

Die Mobilität der ukrainischen Staatsbürger zwischen NGCAs und GCAs sollte durch Unterstützung einer unkomplizierten, sicheren und transparenten Regelung für den Austausch zwischen den NGCAs und GCAs an Checkpoints entlang der Kontaktlinie erleichtert werden. Die gilt insbesondere für die Wiedereröffnung von Checkpoints und die Eröffnung weiterer Kontrollstellen in der Region Luhansk (bei Schaste und Zolotoe).

\section{3) Transparenz und Kommunikation}

Mehrere Befragte aus den GCAs, aus den NGCAs, aus der Flüchtlingsbevölkerung und aus Russland haben zu erkennen ge- geben, dass sie ihre wenigen Informationen zum Inhalt und zu den Abläufen des Minsker Prozesses vor allem aus informellen Kanälen beziehen.

\section{Empfehlungen}

\section{a) an die OSZE sowie die ukrainische und die russische Regierung}

Es sollten Mechanismen für systematische und regelmäßige Kommunikation entwickelt werden, um Informationen über den Minsker Prozess, den Fortschritt der Verhandlungen und die laufenden Bemühungen der TKG mit der Öffentlichkeit, inklusive ukrainischen, russischen, und internationalen Zielgruppen, zu teilen.

\section{b) an die ukrainische Regierung}

Es sollte offen und transparent kommuniziert werden, dass der Sonderstatus und die Autonomie der NGCAs Übergangscharakter haben, wie im Minsker Prozess vorgesehen. Dies würde helfen, gesellschaftliche Spannungen und Polarisierung zu verringern. Kleine und temporäre Schritte sind hierbei erfolgversprechender als eine große Gesamtlösung.

\section{c) an die russische Regierung und die De- facto-Behörden in den NGCAs}

Die Einrichtung eines offenen Kommunikationsraums für ukrainische und in- 
ternationale Medien in den NGCAs sollte gefördert werden (inklusive Verfügbarkeit von digitalen, analogen und Kabelformaten für ukrainische und internationale Sendungen).

\section{Notizen}

1 Die Studie „The Minsk Process as Perceived from Within", durchgeführt von Forscher*innen aus vom Konflikt betroffenen Gebieten der Ukraine, Russland und der Schweiz (darunter auch die Autorinnen dieses Beitrags), ist im Rahmen der transnationalen Dialog-Plattform Women's Initiatives for Peace in Donbas (WIPD) entstanden; mehr Informationen dazu s. Dana Jirouš, „Red lights and diapraxis“, FriEnt Website, TJ Blog, 15 November 2019, https://www.f rient.de/en/blogdata/tj-blog/red-lights-a nd-diapraxis?fbclid=IwAR03ALYQC59 5VNxMpo0UV6VYAaYONdIWwOqb VQU1FR65YsnRrvEI6OhevY. WIPD verdanken wir konzeptuelle Unterstützung und Kontakte zu Teilnehmer*innen aus allen Konfliktparteien. Darüber hinaus wurde das Forschungsteam durch das Center for Governance and Culture an der Universität St. Gallen (Schweiz) konzeptionell und finanziell unterstützt, auBerdem durch das Programm „Ukraine Calling", die Robert-Bosch-Stiftung und das deutsche Auswärtige Amt. Ein herzlicher Dank geht auch an die externen Gutachter*innen dieses Beitrags für ihre nützlichen Kommentare.

2 Roger Fisher/William Ury, Getting to Yes: Negotiating Agreement Without Giving In, Boston: Houghton Mifflin, 1981.

Es war nicht das Ziel der Studie, quantitative Informationen zu sammeln oder Mengenverhältnisse zu rekonstruieren. Sie konzentrierte sich vielmehr auf die Analyse der Narrative. Diese Narrative wurden aus thematisch codierten Inter- views abgeleitet und dann mit geografisch und demografisch codierten Gruppen von Interviewpartner*innen verglichen. Die Studie konzentrierte sich auf keine spezielle gesellschaftliche Gruppe und basierte auf freiwilliger Teilnahme. Wahrscheinlich hatten auch aus diesem Grund ca. zwei Drittel der Interviewten eine höhere Ausbildung, waren die meisten im Alter zwischen 35 und 55, und Frauen im Vergleich zu Männern leicht überrepräsentiert (57\%).

4 Siehe Office of the United Nations High Commissioner for Human Rights, „Report on the human rights situation in Ukraine: 16 November 2019 to 15 February 2020“, Februar 2020, https://www.oh chr.org/Documents/Countries/UA/29thR eportUkraine_EN.pdf.

5 Ministry of Social Policy of Ukraine, Official Website, 12. Mai 2020, https://www .msp.gov.ua/news/18640.html.

6 Maria Litvinova, „Mama posadila menya $\mathrm{v}$ poezd $\mathrm{v}$ Belgorod, a na sleduyushchiy den' $\mathrm{v}$ zdanie vokzala popal snyaryad“ („Meine Mutter setzte mich in einen Zug nach Belgorod, und am nächsten Tag traf eine Granate das Bahnhofsgebäu-

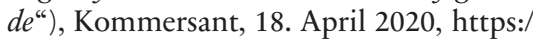
/www.kommersant.ru/doc/4323849.

7 Letztere werden auch als „Certain Areas of Donetsk and Luhansk Regions" (CADLRs) bezeichnet.

8 Siehe: RFE/RL Ukrainian Service, „Ukrainian lawmakers extend Donbas special status law until end of 2020", RFE/RL, 12. Dezember 2019, https://ww w.rferl.org/a/ukrainian-lawmakers-extend -donbas-special-status-law-until-end-of-20 20/30321863.html.

9 Johan Galtung, Peace: Research - Education - Action. Essays in Peace Research I, Copenhagen: Ejlers, 1975.

10 Als positives Beispiel ist in diesem $\mathrm{Zu}-$ sammenhang der Blog über laufende Aktivitäten im Minsker Prozess auf der Webseite der ukrainischen Präsidialver- 
waltung zu nennen: „TCG meeting: OSCE noted sustainable regime of silence for 80 days, emphasizing the need for its further observance“, President of
Ukraine Official Website, 14 October 2020, https://www.president.gov.ua/en/ news/zasidannya-tkg-obsye-vidznachila-st alij-rezhim-tishi-vprodov-64589. 
